\title{
Self-organizing Dynamics for Optimization
}

\author{
Stefan Boettcher \\ Physics Department, Emory University, Atlanta, Georgia 30322-2430, USA
}

\begin{abstract}
Motivated by noise-driven cellular automata models of selforganized criticality (SOC), a new paradigm for the treatment of hard combinatorial optimization problems is proposed. An extremal selection process preferentially advances variables in a poor local state. The ensuing dynamic process creates broad fluctuations to explore energy landscapes widely, with frequent returns to near-optimal configurations.
\end{abstract}

\section{Motivation from Self-organized Criticality}

The study of certain randomly-driven, dissipative automata model with local threshold dynamics has provided a plausible view of many self-organizing processes ubiquitous in Nature [1]. Most famously, the Abelian sandpile model [3] has been used to describe the statistics of earthquakes [1]. Another variant is the Bak-Sneppen model (BS) [2], in which variables are updated sequentially based on a global threshold condition. It provides an explanation for broadly distributed extinction events [21] and the "missing link" problem [14]. Complexity in these SOC models emerges purely from the dynamics, without fine-tuning of parameters, as long as driving is slow and the ensuing avalanches are fast.

In the BS, "species" are located on the sites of a lattice, and have an associated "fitness" value between 0 and 1 . At each time step, the one species with the smallest value (poorest degree of adaptation) is selected for a random update, having its fitness replaced by a new value drawn randomly from a flat distribution on the interval $[0,1]$. But the change in fitness of one species impacts the fitness of interrelated species. Therefore, all of the species at neighboring lattice sites have their fitness replaced with new random numbers as well. After a sufficient number of steps, the system reaches a highly correlated state known as self-organized criticality (SOC) [3]. In that state, almost all species have reached a fitness above a certain threshold. These species, however, possess punctuated equilibrium [14]: only one's weakened neighbor can undermine one's own fitness. This coevolutionary activity gives rise to chain reactions called "avalanches", large fluctuations that rearrange major parts of the system, potentially making any configuration accessible.

We have described [7] the spatio-temporal complexity of the BS by a delay equation for the evolution of activity $P(r, t)$ after a perturbation at $(r=0, t=0)$,

$$
\partial_{t} P(r, t)=\nabla_{r}^{2} P(r, t)+\int_{t^{\prime}=0}^{t} d t^{\prime} V\left(t-t^{\prime}\right) P\left(r, t^{\prime}\right), \quad V(t) \sim t^{-\alpha},
$$


which has the solution $P(r, t) \sim \exp \left\{-C\left(r^{D} / t\right)^{\frac{1}{D-1}}\right\}$ with $D=2 /(\alpha-1)$. Thus, it is the memory of all previous events that determines the current activity.

\section{Extremal Optimization}

Although coevolution may not have optimization as its exclusive goal, it serves as a powerful paradigm. We have used it as motivation for a new approach to approximate hard optimization problems $[8,9]$. The heuristic we have introduced, called extremal optimization (EO), follows the spirit of the BS, updating those variables which have among the "worst" values in a solution and replacing them by random values without ever explicitly improving them. The resulting heuristic explores the configuration space $\Omega$ widely with frequent returns to near-optimal solutions, without fine-tuning of parameters.

To introduce EO, let us consider a spin glass [20] as a specific example of an NP-hard optimization problem. It consists of a $d$-dimensional hyper-cubic lattice of length $L$ with periodic boundary conditions, with a spin variable $x_{i} \in\{-1,1\}$ at each site $i, 1 \leq i \leq n\left(=L^{d}\right)$. A spin is connected to each of its nearest neighbors $j$ via a bond variable $J_{i j} \in\{-1,1\}$, assigned at random. We minimize

$$
C(S)=H(\mathbf{x})=-\sum_{\langle i, j\rangle} J_{i j} x_{i} x_{j}
$$

as (Hamiltonian) cost function, the sum extending over all nearest-neighbor pairs of spins. Due to frustration [20], ground states $S_{\min }$ are hard to find for $d>2$.

To find near-optimal solutions for a particular optimization problem, EO performs a neighborhood search on a single configuration $S \in \Omega$. As in the spin problem in Eq. (2), $S$ consists of a large number $n$ of variables $x_{i}$. We assume that each $S$ possesses a neighborhood $N(S)$ that rearranges the state of merely a small number of the variables. This is a characteristic of a local search, in contrast to a genetic algorithm, say, where cross-overs may effect $O(n)$ variables on each update. The cost $C(S)$ is assumed to consist of the individual cost contributions, or "fitnesses", $\lambda_{i}$ for each variable $x_{i}$. The fitness of each variable assesses its contribution to the total cost and typically the fitness $\lambda_{i}$ depends on the state of $x_{i}$ in relation to connected variables. For example, for the Hamiltonian in Eq. (2), we assign to each spin $x_{i}$ the fitness

$$
\lambda_{i}=x_{i} \sum_{<, j>} J_{i j} x_{j}, \quad C(S)=-\sum_{i=1}^{n} \lambda_{i} .
$$

Each spin's fitness thus corresponds to (the negative of) its local energy contribution to the overall energy of the system. In similarity to the BS, EO then proceeds through a neighborhood search of $\Omega$ by sequentially changing variables with "bad" fitness on each update, for instance, via single spin-flips. After each update, the fitnesses of the changed variable and of all its connected neighbors are reevaluated according to Eq. (3).

The algorithm operates on a single configuration $S$ at each step. Each variable $x_{i}$ in $S$ has a fitness, of which the "worst" is identified. This ranking of the 
variables provides the only measure of quality on $S$, implying that all other variables are "better" in the current $S$. In the move to a neighboring configuration, typically only a small number of variables change state, so only a few connected variables need to be re-evaluated [step (2a)] and re-ranked [step (2b)]. In detail:

1. Initialize configuration $S$ at will; set $S_{\text {best }}:=S$.

2. For the "current" configuration $S$,

(a) evaluate $\lambda_{i}$ for each variable $x_{i}$,

(b) find $j$ satisfying $\lambda_{j} \leq \lambda_{i}$ for all $i$, i.e., $x_{j}$ has the "worst fitness",

(c) choose $S^{\prime} \in N(S)$ such that $x_{j}$ must change,

(d) accept $S:=S^{\prime}$ unconditionally,

(e) if $C(S)<C\left(S_{\text {best }}\right)$ then set $S_{\text {best }}:=S$.

3. Repeat at step (2) as long as desired.

4. Return $S_{\text {best }}$ and $C\left(S_{\text {best }}\right)$.

There is no parameter to adjust for the selection of better solutions. It is the memory encapsulated in the ranking that directs EO into the neighborhood of increasingly better solutions. Like BS, those "better" variables possess punctuated equilibrium: their memory only get erased when they happen to be connected to one of the variables forced to change. On the other hand, in the choice of move to $S^{\prime}$, there is no consideration given to the outcome of such a move, and not even the worst variable $x_{j}$ itself is guaranteed to improve its fitness. Accordingly, large fluctuations in the cost can accumulate in a sequence of updates. Merely the bias against extremely "bad" fitnesses produces improved solutions.

Tests have shown that this basic algorithm is very competitive for optimization problems [8]. But in cases such as the single spin-flip neighborhood for the spin Hamiltonian, focusing on only the worst fitness [step (2b)] leads to a deterministic process, leaving no choice in step (2c): If the "worst" spin $x_{j}$ has to flip and any neighbor $S^{\prime}$ differs by only one flipped spin from $S$, it must be $S^{\prime}=\left(S / x_{j}\right) \cup\left\{-x_{j}\right\}$. This deterministic process inevitably will get stuck near some poor local minimum. To avoid these "dead ends" and to improve results [8], we introduce a single parameter into the algorithm. Ranking all $x_{i}$ according to fitness $\lambda_{i}$, i.e., we find a permutation $\Pi$ of the variable labels $i$ with

$$
\lambda_{\Pi(1)} \leq \lambda_{\Pi(2)} \leq \ldots \leq \lambda_{\Pi(n)} .
$$

The worst variable $x_{j}$ [step (2b)] is of rank $1, j=\Pi(1)$, and the best variable is of rank $n$. Now, consider a scale-free probability distribution over the ranks $k$,

$$
P_{k} \propto k^{-\tau}, \quad 1 \leq k \leq n,
$$

for a fixed value of the parameter $\tau$. At each update, select a rank $k$ according to $P_{k}$. Then, modify step (2c) so that $x_{j}$ with $j=\Pi(k)$ changes its state.

For $\tau=0$, this " $\tau$-EO" algorithm is simply a random walk through $\Omega$. Conversely, for $\tau \rightarrow \infty$, it approaches a deterministic local search, only updating the lowest-ranked variable, and is bound to reach a dead end (see Fig. 1). However, 

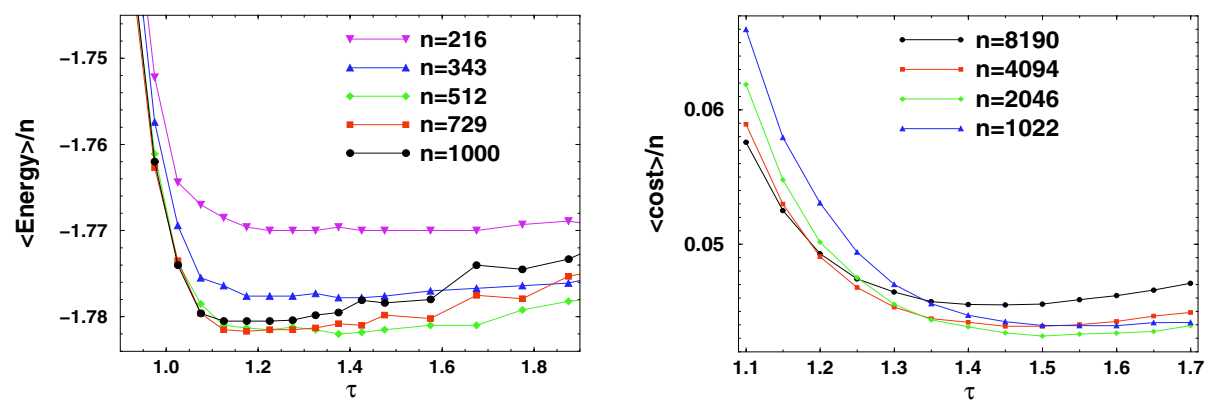

Fig. 1. Plot of costs obtained by EO for a $\pm J$ spin glass (left) and for graph bipartitioning (right), both as a function of $\tau$. For each size $n$, a number of instances were generated. For each instance, 10 different EO runs were performed at each $\tau$. The results were averaged over runs and instances. Although both problems are quite distinct, in either case the best results are obtained at a value of $\tau$ with $\tau \rightarrow 1^{+}$for $n \rightarrow \infty$

for finite values of $\tau$ the choice of a scale-free distribution for $P_{k}$ in Eq. (5) ensures that no rank gets excluded from further evolution while maintaining a bias against variables with bad fitness. In all problems studied, a value of

$$
\tau-1 \sim 1 / \ln n \quad(n \rightarrow \infty)
$$

seems to work best $[9,10]$. We have studied a simple model problem for which the asymptotic behavior of $\tau$-EO can be solved exactly [6]. The model reproduces Eq. (6) exactly in cases where the model develops a "jam" amongst its variables, which is quite a generic feature of frustrated systems.

In Fig. 2 we show the range of states that are sampled during a typical run of EO, here for a spin-glass instance with $n=7^{3}$ and for the image alignment problem [19]. Starting with a random initial condition, for the first $O(n)$ update steps EO establishes local order, leading to a rapid decrease in the energy. After
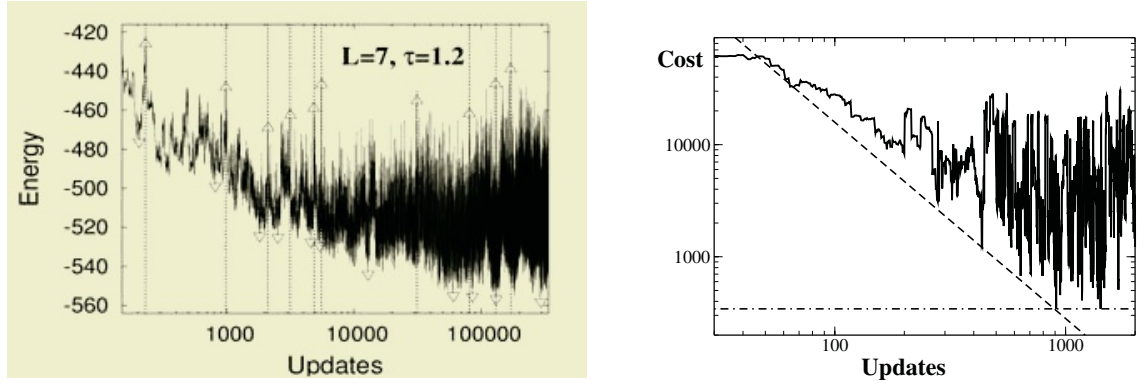

Fig. 2. Plots of the range of states attained by EO during single run on a particular instance (of an $L=7$ cubic spin glass (left) and of the image alignment problem [19] (right). After an initial transient, the ultimate "steady state" is reached in which EO fluctuates widely through near-optimal configurations, obtaining increasingly better energy records $(\nabla)$ while scaling ever higher barriers $(\triangle)$ 
that EO searches through a wide band of states with frequent returns to nearoptimal configurations.

\section{Numerical Results for EO}

In the few years since we first proposed (EO) as a general purpose heuristic for some of the hardest combinatorial optimization problems [8], ample evidence has been provided for its practicality [9, 10,11]. Our own studies have focused on demonstrating elementary properties of EO in a number of implementations for classic NP-hard combinatorial problems such as graph bipartitioning [8,10], 3-coloring [11], spin glasses [9], and the traveling salesperson [8]. Several other researchers have picked up on our initial results, and have successfully applied EO to problems as diverse as pattern recognition [19], signal filtering of EEG noise [24], artificial intelligence [18], and $3 d$ spin-glass models $[12,23]$. Comparative studies have shown that EO holds significant promise to provide a new, alternative approach to approximate many intractable problems $[8,12,18]$.

\subsection{Results on Spin Glasses}

To gauge $\tau$-EO's performance for larger $3 d$-lattices, we have run our implementation also on two instances, toruspm3-8-50 and toruspm3-15-50, with $n=512$ and $n=3375$, considered in the $7 t h$ DIMACS challenge for semi-definite problems ${ }^{1}$. The best available bounds (thanks to F. Liers) established for the larger instance are $H_{\text {lower }}=-6138.02$ (from semi-definite programming) and $H_{\text {upper }}=-5831$ (from branch-and-cut). EO found $H_{\mathrm{EO}}=-6049$ (or $H / n=-1.7923$ ), a significant improvement on the upper bound and already lower than $\lim _{n \rightarrow \infty} H / n \approx$ $-1.786 \ldots$ found in Ref. [9]. Furthermore, we collected $10^{5}$ such states, which roughly segregate into three clusters with a mutual Hamming distance of at least 100 distinct spins; though at best a small sample of the $\approx 10^{73}$ ground states expected [15]! For the smaller instance the bounds given are -922 and -912 , while EO finds -916 (or $H / n=-1.7891$ ) and was terminated after finding $10^{5}$ such states. While this run (including sampling degenerate states) took only a few minutes of CPU (at $800 \mathrm{MHz}$ ), the results for the larger instance required about 16 hours.

More recently, we have combined EO with reduction methods for sparse graphs $[4,5]$. These reductions strip graphs of all low-connected variables $(\alpha \leq$ 3 ), thereby eliminating many entropic barriers that tend to bog down local searches [22]. Along the way, the rules allow for an accounting of the exact ground-state energy and entropy, and even of the approximate overlap distribution [5]. The "remainder" graph is subsequently handled efficiently with EO. With such a meta-heuristic approach, for example, we have been able to determine the defect energy distribution [13] for $d=3, \ldots, 7$ dimensional spin

\footnotetext{
${ }^{1}$ http://dimacs.rutgers.edu/Challenges/Seventh/
} 
glasses, bond-diluted to just above their percolation point, with great accuracy for lattices up to $L=30$ [4]. As one result, we reduced the error on the stiffness exponent in $d=3, y_{d=3}=0.240(5)$, from $20 \%$ to about $2 \%$. This fundamental exponent describes the energetic cost $\Delta E$ of perturbations (here, induced interfaces) of size $L, \sigma(\Delta E) \sim L^{y}$.

Currently, we are using this meta-heuristic to explore the (possible) onset of replica symmetry breaking (RSB) for sparse mean-field and lattice models just above percolation. So far, we have only some preliminary data for Spin glasses on random graphs. In this model at connectivities near percolation $\alpha \approx \alpha_{p}=1$, many spins may be entirely unconnected while a finite fraction is sufficiently connected to form a "giant component" in which interconnected spins may become overconstrained. There the reduction rules allow us to reduce completely a statistically significant number of graphs with up to $n=2^{18}$ spins even well above $\alpha_{p}$, since even higher-connected spins may become reducible eventually after totally reducible substructures (trees, loops, etc) emanating from them have been eliminated. At the highest connectivities reached, even graphs originally of $n=2^{18}$ had collapsed to at most 100 irreducible spins, which EO easily optimized.
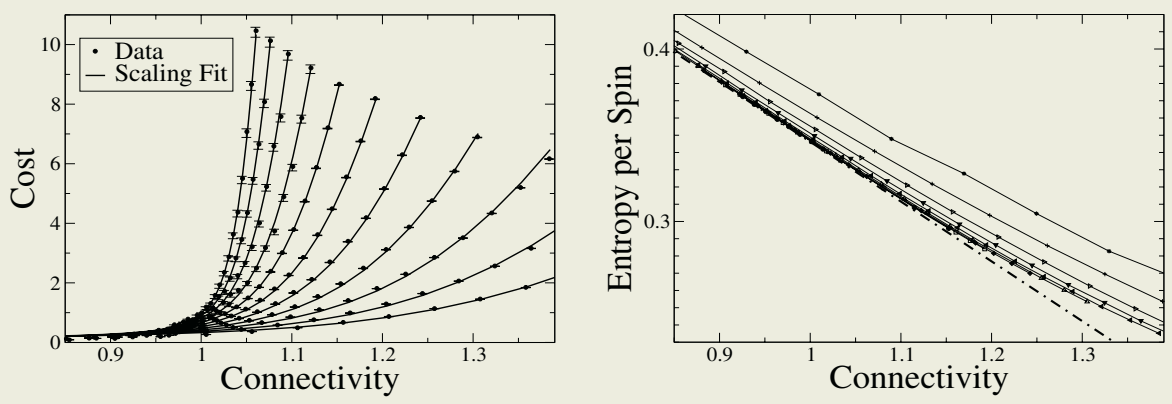

Fig. 3. Plot (left) of the cost and (right) of the entropy per spin, as a function of connectivity $\alpha$ for random graphs of size $n=2^{8}, 2^{9}, \ldots, 2^{18}$. For increasing $n$, the cost approaches a singularity at $\alpha_{\text {crit }}=1.003(9)$, as determined from a finite-size scaling fit (lines on left) to $\langle C\rangle(\alpha, n) \sim n^{\delta} f\left[\left(\alpha-\alpha_{\text {crit }}\right) n^{1 / \nu}\right]$. The fit predicts also $\delta=0.11(2)$ and $\nu=3.0(1)$. The entropy per spin quickly converges to $\approx(1-\alpha / 2) \ln 2$ (dashed line), exact for $\alpha<\alpha_{\text {crit }}=1$, continues unaffected through the transition, but deviates from that line for $\alpha>\alpha_{\text {crit }}$

As a result, we have measured the cost of ground states, Eq. (2), as a function of connectivity $\alpha$ on 40000 instances for each size $n=2^{8}, 2^{9}, \ldots, 2^{14}$, and 400 instances for $n=2^{15}, \ldots, 2^{18}$, at each of 20 different connectivities $\alpha$ as shown in Figure 3. We also account exactly for the degeneracy of each instance, which could number up to $\exp \left[0.3 \times 2^{18}\right]$; minuscule compared to all $2^{2^{18}}$ configurations! Not entirely reduced graphs had their entropy determined with EO in our meta-heuristic. Consistent with theory [17], Figure 3 shows that the entropy per spin follows $s \approx(1-\alpha / 2) \ln 2$ for $\alpha<\alpha_{\text {crit }}=1$, then continues smoothly 
through the transition but deviates from that line for $\alpha>\alpha_{\text {crit }}$. Similar data for the overlap-moments [5] may determine the onset of RSB expected for this model.

\subsection{Applications of EO by Others}

The generality of the EO method beyond the domain of spin-glass problems has recently been demonstrated by Meshoul and Batouche [19] who used the EO algorithm as described above successfully on a standard cost function for aligning natural images. Fig. 4 demonstrates the results of their implementation of $\tau$-EO for this pattern recognition problem. Here, $\tau$-EO finds an optimal affine transformation between a target image and its reference image using a set of $n$ adjustable reference points which try to attach to characteristic features of the target image. The crucial role played by EO's non-equilibrium fluctuations in the local search is demonstrated in Fig. 2. The fluctuations in the image alignment problem are amazingly similar to those we have found for spin glasses. As our discussion in Sec. 2 suggests, they are one of the key distinguishing features of EO, and are especially relevant for optimizing highly disordered systems. For instance, Dall and Sibani [12] have observed a significantly broader distribution of states visited - and thus, better solutions found - by $\tau$-EO compared to simulated annealing [16] when applied to the Gaussian spinglass problem.

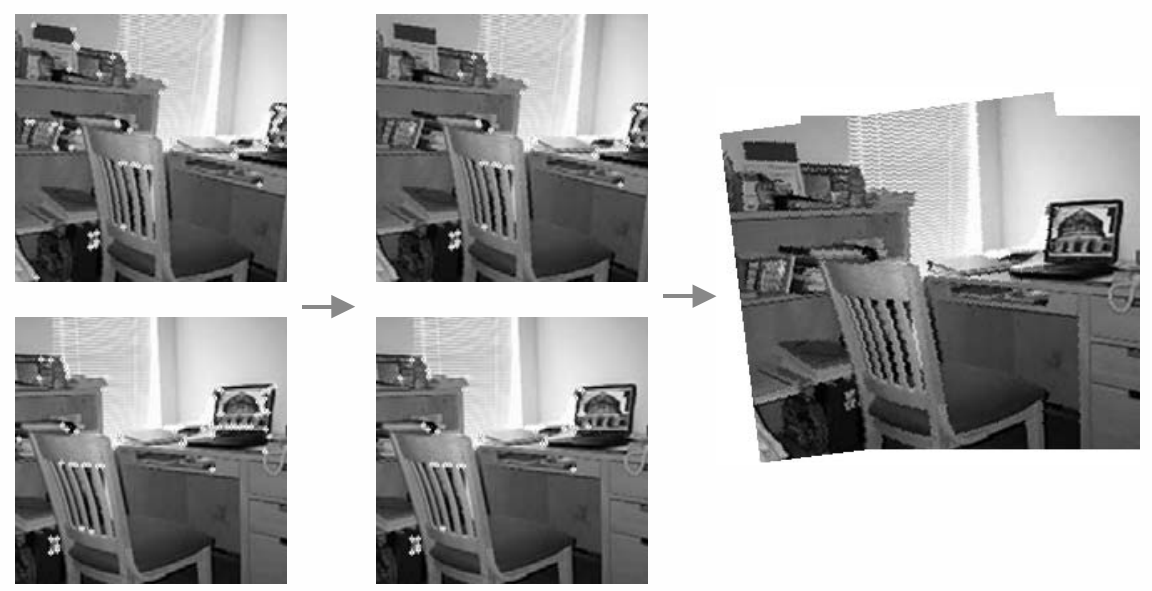

Fig. 4. Application of EO to the image matching problem, after [19]. Two different images of the same scene (top row and bottom row) are characterized by a set of $n$ points assigned by a standard pattern recognition algorithm. Starting from an initial assignment (left, top and bottom), the points are updated according to EO, see also Fig. 2, leading to an optimal assignment (center, top and bottom). This optimal assignment minimizes a cost function for the affine transformation, facilitating an automated alignment of the two images (right). Note that the points move to the part of the scene for which both images overlap. Special thanks to M. Batouche for providing those images 


\section{Acknowledgements}

I would like to thank M. Paczuski, A.G. Percus, and M. Grigni for their collaboration on many aspects of the work presented here. This work was supported under NSF grant DMR-0312510 and Emory's URC.

\section{References}

1. P. Bak, How Nature Works (Springer, New York, 1996).

2. P. Bak and K. Sneppen, Punctuated Equilibrium and Criticality in a simple Model of Evolution, Phys. Rev. Lett. 71, 4083-4086 (1993).

3. P. Bak, C. Tang, and K. Wiesenfeld, Self-Organized Criticality, Phys. Rev. Lett. 59, 381 (1987).

4. S. Boettcher, Low-Temperature Excitations of Dilute Lattice Spin Glasses, Europhys. Lett. 67, 453-459 (2004).

5. S. Boettcher, Reduction of Spin Glasses applied to the Migdal-Kadanoff Hierarchical Lattice, Euro. Phys. J. B 33, 439-445 (2003).

6. S. Boettcher and M. Grigni, Jamming Model for the Extremal Optimization Heuristic, J. Math. Phys. A: Math. Gen. 35, 1109-1123 (2002).

7. S. Boettcher and M. Paczuski, Ultrametricity and Memory in a Solvable Model of Self-Organized Criticality, Physical Review E 54, 1082 (1996).

8. S. Boettcher and A. G. Percus, Nature's Way of Optimizing, Artificial Intelligence 119, 275-286 (2000).

9. S. Boettcher and A. G. Percus, Optimization with Extremal Dynamics, Phys. Rev. Lett. 86, 5211-5214 (2001).

10. S. Boettcher and A. G. Percus, Extremal Optimization for Graph Partitioning, Phys. Rev. E 64, 026114 (2001).

11. S. Boettcher and A. G. Percus, Extremal Optimization at the Phase Transition of the 3-Coloring Problem, Physical Review E 69, 066703 (2004).

12. J. Dall and P. Sibani, Faster Monte Carlo Simulations at Low Temperatures: The Waiting Time Method, Computer Physics Communication 141, 260-267 (2001).

13. K. H. Fischer and J. A. Hertz, Spin Glasses (Cambridge University Press, Cambridge, 1991).

14. S. J. Gould and N. Eldridge, Punctuated Equilibria: The Tempo and Mode of Evolution Reconsidered, Paleobiology 3, 115-151 (1977).

15. A. K. Hartmann, Ground-state clusters of two-, three- and four-dimensional +-J Ising spin glasses, Phys. Rev. E 63, 016106 (2001).

16. S. Kirkpatrick, C. D. Gelatt, and M. P. Vecchi, Optimization by simulated annealing, Science 220, 671-680 (1983).

17. M. Leone, F. Ricci-Tersenghi, and R. Zecchina, Phase coexistence and finite-size scaling in random combinatorial problems, J. Phys. A. 34, 4615 (2001).

18. M. B. Menai and M. Batouche, Approximate solution of Max-SAT problem using Extremal Optimization heuristic, Journal of Automated Reasoning, (to appear).

19. S. Meshoul and M. Batouche, Robust Point Correspondence for Image Registration using Optimization with Extremal Dynamics, Lect. Notes Comput. Sc. 2449, 330337 (2002).

20. M. Mezard, G. Parisi, and M. A. Virasoro, Spin Glass Theory and Beyond (World Scientific, Singapore, 1987). 
21. D. M. Raup and J. J. Sepkoski, Periodic Extinction of Families and Genera, Science 231, 833-836.

22. F. Ricci-Tersenghi, M. Weigt, and R. Zecchina, Simplest random K-satisfiability problem, Phys. Rev. E 63, 026702 (2001).

23. J.-S. Wang and Y. Okabe, A comparison of extremal optimization with flathistogram dynamics for finding spin-glass ground states, J. Phys. Soc. Jpn. 72, 1380-1383 (2003).

24. E. Yom-Tov, A. Grossman, and G. F. Inbar, Movement-related potentials during the performance of a motor task I: The effect of learning and force, Bio. Cybernatics 85, 395-399 (2001). 